\title{
La tâche pédagogique au service du développement des savoir-faire langagiers et professionnels. Exemple de français des affaires
}

\author{
Didactic tasks and development of language \\ and vocational skills. The French business example
}

\begin{abstract}
Task Based Learning / Teaching focuses on the action dimension of language use, i.e., various tasks performed every day that require cooperation with others and the mobilization of language. Language proves to be the most important instrument of action, even if the objective of this action is not necessarily linguistic. The pedagogical tasks used for language teaching for professional purposes illustrate the link between language and socio-professional action: the learning of the formal language system counts as much as the content of the domain of work. On these theoretical assumptions, the aim of this paper is to examine the degree of integration of linguistic and professional knowledge and skills into tasks designed for the teaching of business French. We will analyse the examples of pedagogical tasks in order to demonstrate to what extent they allow us to teach interdisciplinary content and foreign language.
\end{abstract}

KEYWORDS: language for specific purposes, to act, language skills, vocational skills, task.

MOTS-CLÉS: Langue sur objectifs spécifiques, agir, savoir-faire langagiers, savoir-faire professionnels, tâche.

La vie de chacun requiert l'accomplissement d'actions de nature diverse, p.ex. préparer le repas, répondre à un mél, payer la facture d'électricité, candidater à des emplois, rédiger un article scientifique etc. En fonction de la nature et du contexte des actions, ces dernières contraignent l'individu à recourir à des modes d'action déterminés et à mobiliser, à chaque fois, les diverses ressources les mieux adaptées à l'objectif visé. Se pose alors la question de savoir si ces ressources sont disponibles pour l'agent et à quel niveau, ce qui renvoie directement à la question de la possibilité ou de l'impossibilité de l'accomplissement de l'action par celui qui agit : Sais-je le faire ou non ? Suis-je capable de le faire ou non ? La question de savoirs nécessaires pour la résolution d'une action s'avère décisive pour que l'agent décide consciemment de l'entreprendre et la mener à bien. Il importe alors de s'interroger sur la façon et les modalités de l'appropriation des savoirs et savoir-faire qui rendent l'action possible. 
Dans ce texte, nous avons choisi d'entreprendre une telle réflexion par rapport à l'acquisition des savoirs nécessaires à l'accomplissement des actions langagières insérées dans le contexte professionnel. Plus concrètement, il s'agit d'analyser un certain nombre de tâches pédagogiques du point de vue de leur contenu (c'est-à-dire des savoirs et savoir-faire langagiers et professionnels) ainsi que de démontrer comment et dans quelle mesure elles préparent l'apprenant à réaliser les actions langagières (en français) dans le milieu du travail.

\section{APPRENDRE POUR AGIR ET AGIR POUR APPRENDRE}

Les savoirs d'un individu sont nombreux et multiformes : ils résument les acquis de la trajectoire scolaire et professionnelle de chacun ; ils traduisent aussi les savoirs accumulés dans la sphère privée, que ce soit dans la famille ou plus généralement dans la vie hors travail. Le potentiel est la condition de l'action et l'action est une des conditions du potentiel. La mobilisation des savoirs est tributaire des processus d'apprentissage développés tout au long de la vie professionnelle et sociale, en vue d'améliorer le potentiel des individus (De Terssac 1996 : 237).

Puisque, dans ce texte, nous envisageons l'acquisition des savoirs dans les situations d'apprentissage, notre intérêt est de nous concentrer sur celles qui privilégient un apprentissage actionnel. C'est pourquoi nous orientons davantage notre réflexion sur la perspective actionnelle (ou par tâches) recommandée depuis 2001 par le Conseil de l'Europe et particulièrement son document-phare Cadre Européen Commun de Référence pour les Langues (désormais CECRL). Son idée de départ est de soutenir et de promouvoir des méthodes d'enseignement / apprentissage censées aider les apprenants à « se forger les savoirs, savoir-faire et attitudes dont ils ont besoin pour acquérir davantage d'indépendance dans la réflexion et dans l'action afin de se montrer plus responsables et coopératifs dans les relations à autrui » (CECRL $2001: 4)$.

Bien qu'associée au CECRL et promue vivement par ce dernier, l'idée de la subordination de l'apprentissage à l'action n'est pas l'apanage de la pensée didactique de la dernière vingtaine d'années. Nous trouvons déjà ses diverses facettes dans les conceptions de Dewey (« Learning by doing »), de Freinet (" pédagogie du projet») ou de Bruner («apprentissage par découverte »). La version anglo-saxonne de l'apprentissage par tâches («Task-Based Learning ») s'appuie également sur les principes actionnels similaires. Toutes les conceptions et théories susmentionnées ont un dénominateur commun :

soumettre l'apprentissage à la logique de la production en supprimant le hiatus entre apprendre et agir et en inversant le rapport habituel : au lieu d'apprendre d'abord 
en un temps séparé pour faire ensuite selon un modèle d'application, on pose que l'on apprend parce que l'on fait et par ce que l'on fait. Dans cette perspective, le produit que l'on vise est considéré comme une masse de savoirs investis [...] qui sont requis tout soudain dans le développement de la tâche, construits dans l'évolution de l'objet, immédiatement investis en lui, introduits dans le processus sous forme de problèmes à résoudre pour continuer (Halté 1982 : 21).

Nous en concluons que les activités d'apprentissage doivent ressembler - du point de vue de la structure et de l'objectif - aux actions sociales et / ou professionnelles censées contribuer à l'activation du potentiel compétentiel des agents et à la formation plus ou moins définitive de leurs savoirs, savoir-faire et attitudes quel que soit le domaine de l'usage de la langue.

\section{LE POTENTIEL DE LA TÂCHE POUR L'ENSEIGNEMENT DU FLE / FOS'}

\section{L'idée que le CECRL (2001) accentue fortement consiste à considérer}

l'usager et l'apprenant d'une langue comme des acteurs sociaux ayant à accomplir des tâches (qui ne sont pas seulement langagières) dans des circonstances et un environnement donnés, à l'intérieur d'un domaine d'action particulier. Si les actes de parole se réalisent dans des activités langagières, celles-ci s'inscrivent elles-mêmes à l'intérieur d'actions en contexte social qui seules leur donnent leur pleine signification (CECRL 2001 : 15).

Il en résulte que les situations d'apprentissage sont jugées comme l'occasion d'accomplissement des tâches à l'image des actions que l'on réalise en société hors milieu scolaire. La tâche - notion-clé de la perspective actionnelle - équivaut à l'action et devient ainsi son synonyme : « est définie comme tâche toute visée actionnelle que l'acteur se présente à parvenir à un résultat donné en fonction d'un problème à résoudre, d'une obligation à remplir, d'un but qu' on s'est fixé » (CECRL 2001 : 194).

L'omniprésence des actions / tâches de diverse nature dans la vie quotidienne renforce le lien entre l'apprentissage et l'action et donne du sens à l'apprentissage :

les tâches sont ces mille et une petites choses que l'on mène à bien tous les jours, pour soi-même ou pour autrui, gratuitement ou pour de l'argent, et que l'on fait

${ }^{1}$ Même si le terme de « Français sur Objectifs Spécifiques » renvoie à une démarche pédagogique bien particulière et spécifique (cf. Mangiante \& Parpette 2004), pour le besoin de ce texte, nous l'utiliserons par rapport à l'ensemble de situations d'enseignement / apprentissage qui visent à enseigner / apprendre la langue à des fins professionnelles. 
quelquefois parce que l'on y est obligé. Se brosser les dents, écrire un court essai, emprunter un livre : ce sont autant de tâches. Un grand nombre, sinon la majorité des tâches que l'on effectue, demandent de recourir au langage sous une forme ou sous une autre (Devitt 2002 : 100).

D'où l'utilité de ce type d'activité pédagogique dans l'enseignement / apprentissage des langues. Reste cependant à observer que, dans la tâche, la langue s'attribue un statut particulier : elle est un outil d'action et non l'objectif de celle-ci. Il en résulte que, pour l'apprenant, il est question d'accomplir la tâche grâce à la mobilisation des ressources linguistiques correctes et adéquates au contexte ; l'apprentissage du système formel de la langue y compte autant que le contenu extralinguistique de la tâche.

\section{La tâche}

inclut la gestion du sens en lien direct avec le monde réel, avec un objectif défini, et le résultat pragmatique obtenu prime sur la performance langagière. Du fait de son caractère réaliste, de son enracinement dans le concret, de son orientation vers le résultat, la tâche présente de fortes affinités avec l'enseignement des langues à visées professionnelles, orienté vers la poursuite d'objectifs spécifiques et la recherche d'efficacité (Narcy-Combes \& Narcy-Combes 2007 : 75-76).

Les caractéristiques de la tâche évoquées ci-avant traduisent sa forte position dans l'enseignement du FOS qui, depuis toujours, privilégie les formes actives d'enseignement / apprentissage.

Le statut modifié de la langue dans la tâche (où la langue équivaut à un outil d'action) par rapport aux activités communicatives (où la langue est déterminée comme objectif de la communication) attire l'attention sur le mode de fonctionnement et d'usage de la langue dans le milieu professionnel. Mobiliser la langue au service des tâches professionnelles suppose de s'en servir comme instrument $\mathrm{d}^{\prime}$ action dont le statut n'est pas stable et dont les formes varient d'une situation à l'autre. Lacoste (2001 : 33) observe qu'il y a

la parole dans l'activité que le contexte même suscite et transforme, la parole comme activité lorsque le travail est d'ordre langagier, le «faire » équivaut donc au "dire », la parole sur l'activité qui se situe au niveau « méta » et contraint à une attitude réflexive, la parole comme garante de l'identité personnelle et le signe d'appartenance à un groupe cohérent.

Il en résulte que le langage au travail revêt des formes variées : "à l'oral, il s'accompagne du non-verbal (intonation, regard, mimique, geste); à l'écrit, il circule sous formes officielles et standardisées (lettre, compte rendu, procèsverbal), mais aussi en incarnant les formes de 'post-it', fiches, formulaires, codes, 
listes, graphiques, images, écrans etc. » (Sowa 2011 : 130). L'usage de la langue de même que sa compréhension dépendent du contexte inhérent à la profession et des circonstances immédiates, puisque la façon de dire s'inscrit dans un univers professionnel donné (personnes, objets, institutions, événements, lieux etc.), cadré par les instances administratives et soumis à des régulations sociales.

Les tâches pédagogiques rendent parfaitement compte de l'utilisation particulière de la langue par des locuteurs professionnels dans le cadre d'une communication spécialisée et à travers des discours spécialisés que les individus mettent au service des actions de travail accomplies dans certaines conditions de leur pratique professionnelle. En tant qu'activité d'apprentissage exigeant la mobilisation de diverses compétences, orientée sur un thème à l'intérieur d'un domaine, insérée dans un contexte et des conditions déterminés, recourant à diverses activités langagières et consistant à traiter divers textes, la tâche peut facilement être exploitée pour préparer l'apprenant à réaliser des actions professionnelles nécessitant l'utilisation de la langue. Les caractéristiques susmentionnées de la tâche donnent la possibilité de travailler différents aspects des savoirs et savoirfaire langagiers et professionnels, tels que p.ex. le contenu thématique d'un domaine de référence, les normes socio-professionnelles d'usage de la langue en usage dans une situation donnée, les genres de textes et discours en usage professionnel, les ressources linguistiques nécessaires etc. (cf. Bronckart 2001). Ces différents éléments de compétence peuvent être abordés grâce à différents types de tâches que le CECRL propose à mettre en place, à savoir :

- « les tâches 'cibles' ou de 'répétition' ou 'proches de la vie réelle' [...] choisies en fonction des besoins de l'apprenant en dehors du milieu scolaire,

- les tâches de nature plus spécifiquement 'pédagogique' fondées sur la nature sociale et interactive 'réelle' et le caractère immédiat de la situation de classe,

- les activités 'méta-communicatives' » (CECRL 2001 :121) qui visent à fournir aux apprenants les éléments de la langue nécessaires pour l'accomplissement de la tâche finale.

La typologie des tâches ${ }^{2}$ présentée ci-avant révèle à la fois les disparités dans la structure des tâches et dans les objectifs visés. Les activités méta-communicatives correspondent aux exercices de langue (de vocabulaire, de grammaire, d'orthographe etc.) et visent à transmettre le savoir. Les tâches pédagogiques dont la structure est simple s' orientent vers le perfectionnement des savoir-faire et constituent des étapes intermédiaires pour l'atteinte de l'objectif final. Ce dernier réside dans les tâches cibles dont l'organisation est complexe et qui visent

${ }^{2}$ Pour le besoin de ce texte, nous avons choisi de nous appuyer sur la définition de la tâche et la typologie proposée par le CECRL (2001). Même si les définitions de tâches abondent dans la littérature, mais diffèrent les unes des autres, les termes définis dans le texte du CECRL constituent pour le présent travail la terminologie de référence. 
l'acquisition définitive des compétences. Grâce à la structure différenciée des tâches et la variété des objectifs qu'elles visent, il est possible de les assembler différemment en fonction des besoins d'enseignement / apprentissage. Les emboiter les unes dans les autres, les subordonner les unes aux autres, agencer les plus simples avec les plus complexes, toutes ces opérations permettent de rendre compte des étapes progressives que parcourt l'apprenant à partir de l'appropriation des savoirs (apprentissage des formes) par le perfectionnement des savoir-faire jusqu'à la mobilisation opérationnelle des compétences (usage autonome de la langue au service de l'action).

\section{LES TÂCHES DANS LES MANUELS DE FOS}

Nous avons déjà évoqué l'intérêt des tâches pour l'enseignement du FOS. La présence massive des tâches dans les méthodes de français professionnel en constitue la meilleure preuve. Reste à nous interroger sur la nature des savoirs et savoir-faire abordés par les tâches et à analyser la manière dont les tâches combinent les savoirs relatifs à la langue et au travail. C'est pourquoi nous avons choisi d'examiner trois méthodes de FOS pour des publics en situation professionnelle ou en préparation à la vie active de niveaux A1 et A2 du CERL, à savoir :

- Gillmann, B. 2007. Travailler en français en entreprise. Méthode de français sur objectifs spécifiques. Paris : Didier - niveaux A1 / A2,

- Dubois, A. L. / Tauzin, B. 2013. Objectif Express 1. Le monde professionnel en français. Paris: Hachette - niveaux A1 > A2,

- Rosillo, M. P. / Maccotta, P. / Demaret, M. 2013. Quartier d'affaires. Français professionnel et des affaires. Paris : CLE International - niveau A2.

Le choix de niveau élémentaire nous permettra de montrer - nous l'espérons que l'intégration des contenus langagiers et professionnels est possible dès les étapes initiales de l'apprentissage d'une langue.

La structure adoptée par la méthode Travailler en français en entreprise (désormais $\mathrm{TeFe}$ ) vise à travailler d'abord les éléments de langue et de communication qui seront investis tous ensemble dans la résolution de la tâche finale (appelée par la méthode « étude de cas »). Le travail des apprenants est ainsi initié par les activités communicatives basées sur la compréhension orale et / ou écrite. Celles-ci sensibilisent déjà aux contenus thématiques de l'unité et mettent en scène les aspects linguistiques à aborder. Elles sont suivies des « points de langue » et exercices de langue dont le but est d'abord d'expliciter les questions de langue (vocabulaire, grammaire) et, ensuite, de permettre aux apprenants de s'entraîner à leur utilisation guidée. Cette dernière s'effectue dans le cadre d'exercices structuraux et / ou d'activités de communication (question-réponse, jeu de rôle etc.). L'étude de cas, 
soit la tâche complexe, intervient comme activité finale qui requiert l'utilisation autonome de l'ensemble des éléments travaillés en amont. La structure de l'unité et l'agencement des activités proposées conduisent l'apprenant des situations d'apprentissage vers la réalisation d'une tâche proche de la vie sociale en autonomie.

Outre leur aspect linguistique, les activités et textes proposés sont également porteurs de contenus professionnels (savoir, savoir-faire et savoir-être). Le travail sur les éléments linguistiques est mis en rapport avec un contexte professionnel d'usage de la langue et une action particulière à réaliser individuellement ou en coopération ayant recours à la langue. Pour que notre propos reste concret, prenons l'exemple de l'une des unités contenues dans la méthode de français en question. L'unité « Faire connaissance » aborde l'un des éléments basiques de l'activité professionnelle, à savoir la présentation. Les objectifs prioritaires visent à se présenter en contexte de travail et à faire la connaissance des autres acteurs professionnels ou de partenaires potentiels. Les cartes de visite et badges professionnels renseignent les apprenants sur les éléments inhérents à la présentation professionnelle, tels que, selon le cas, le nom de l'entreprise, la profession, le poste ou la fonction, le service ou le département qui suivent naturellement le prénom et le nom du salarié.

L'expression de ces données n'est pas possible sans éléments de vocabulaire (substantifs exprimant les noms de métiers, postes, fonctions, services ; les adjectifs de nationalités), de grammaire (verbes utilisés pour la présentation «être », «s'appeler » ; féminin et masculin des substantifs et adjectifs de nationalité ; affirmation et négation ; formulation de questions ; prépositions etc.), de prononciation et / ou d'orthographe, de culture professionnelle (s'adresser au travail : salutations, tutoiement, vouvoiement). C'est pourquoi ces éléments sont d'abord travaillés séparément dans le cadre des tâches méta-communicatives du type : "Complétez les phrases ", "Associez les mots de la colonne gauche et les mots de la colonne droite », «Associez les questions aux réponses » et repris ensuite dans les tâches pédagogiques de communication sollicitées par les consignes : «Écoutez X présenter trois ami(e)s. Complétez le tableau », «Lisez l'article et complétez la fiche », «Posez des questions sur ... à votre partenaire. Changez de rôle », «Pensez à trois ami(e)s ou membres de votre famille. Parlez de vous, de votre travail et de leur travail ». Les photographies, qui accompagnent les activités, mettent en scène des salariés en costume-cravate ou en tailleur qui se serrent la main, en attirant ainsi l'attention des apprenants sur des aspects non-verbaux de l'activité professionnelle.

Présentée à la fin de l'unité, la tâche finale vise à intégrer les ressources linguistiques et les activités langagières (production, interaction, médiation) au profit de l'action menée dans un contexte bien particulier :

Vous êtes chargé(e) de communication pour une école de commerce. Vous êtes à un congrès international au Palais de congrès et des expositions de Nice Acropolis. 
Vous rencontrez des personnes pour faire la promotion du programme MBA de votre école (TeFe 2007 : 15).

Elle comporte aussi plusieurs étapes :

Complétez le badge avec des informations sur vous-même. Faites connaissance avec les autres personnes présentes au congrès. Notez les informations sur les personnes rencontrées. Parlez des personnes rencontrées. Choisissez deux personnes intéressées par le programme MBA de votre école. Écrivez un courriel à votre assistante sur ces deux personnes. Donnez l'adresse électronique de chaque personne pour l'envoi d'une documentation par courriel (TeFe 2007 : 15).

Outre la sélection et la mobilisation appropriée des ressources linguistiques, l'apprenant doit également décider de la nature des éléments à réunir sur les candidats potentiels et transmettre ensuite à son collègue pour l'envoi de la documentation.

L'analyse de l'unité 1 du manuel TeFe nous amène à présenter les contenus d'enseignement / apprentissage abordés à différentes étapes et à l'intérieur de divers types de tâches qui, toutes ensemble, sont subordonnées à la tâche finale et contribuent à la résolution de celle-ci de la manière suivante :

Tableau 1. «Faire connaissance » $(\mathrm{TeFe})$ : intégration des savoirs et savoir-faire langagiers et professionnels

\begin{tabular}{|c|c|c|c|c|}
\hline \multirow{2}{*}{$\begin{array}{l}\text { Actes de } \\
\text { parole }\end{array}$} & \multicolumn{2}{|c|}{ Savoirs et savoir-faire linguistiques } & \multirow{2}{*}{$\begin{array}{c}\text { Savoirs } \\
\text { professionnels }\end{array}$} & \multirow{2}{*}{$\begin{array}{l}\text { Savoir-faire pro- } \\
\text { fessionnels / com- } \\
\text { municationnels }\end{array}$} \\
\hline & Lexique & Grammaire & & \\
\hline 1. Se présenter & \multirow{4}{*}{$\begin{array}{l}\text { - } \text { intitulés de } \\
\text { poste, profes- } \\
\text { sions ; } \\
\text { - niveau de } \\
\text { formation ; } \\
\text { - départe- } \\
\text { ments de } \\
\text { l'entreprise } \\
\text { et fonctions ; } \\
\text { - nationalité ; } \\
\text { - pays ; } \\
\text { - état civil. }\end{array}$} & $\begin{array}{l}\text { - Conjuguer les verbes être, } \\
\text { avoir, s'appeler, travailler, faire, } \\
\text { habiter; } \\
\text { - Utiliser le masculin / féminin }\end{array}$ & \multirow{4}{*}{$\begin{array}{l}\text { - intitulés de } \\
\text { postes et fonc- } \\
\text { tions ; } \\
\text { - implantation } \\
\text { des entreprises } \\
\text { (siège social, } \\
\text { filiales / suc- } \\
\text { cursales) ; } \\
\text { - secteur } \\
\text { d'activité ; } \\
\text { - noms des } \\
\text { entreprises } \\
\text { et types } \\
\text { d'activité. }\end{array}$} & \multirow{4}{*}{$\begin{array}{l}\text { - } \text { se présenter ; } \\
\text { - } \text { faire connais- } \\
\quad \text { sance dans le } \\
\text { milieu profes- } \\
\text { sionnel ; } \\
\text { - lire / concevoir } \\
\quad \text { une carte de } \\
\text { visite ; } \\
\text { - lire / concevoir } \\
\quad \text { un badge ; } \\
\text { - rédiger un cour- } \\
\text { riel officiel. }\end{array}$} \\
\hline $\begin{array}{l}\text { 2. Présenter } \\
\text { qqn }\end{array}$ & & $\begin{array}{l}\text { des nationalités, des pays } \\
\text { (le/la), des professions; } \\
\text { - Utiliser les prépositions } \\
\text { (ville, pays, poste) } \\
\text { - c'est... / voici... / voilà... }\end{array}$ & & \\
\hline $\begin{array}{l}\text { 3. S'informer, } \\
\text { interrompre, } \\
\text { faire répé- } \\
\text { ter, } \ldots\end{array}$ & & $\begin{array}{l}\text { Poser des questions sur qqn } \\
\text { - avec une intonation mont- } \\
\text { ante ; } \\
\text { - avec est-ce que... ; } \\
\text { - avec l'inversion ; } \\
\text { - avec où, qui, que, quel(le), } \\
\text { comment... }\end{array}$ & & \\
\hline $\begin{array}{l}\text { 4. Informer, } \\
\text { renseigner }\end{array}$ & & $\begin{array}{l}\text { Répondre aux questions: } \\
\text { - négation / confirmation }\end{array}$ & & \\
\hline
\end{tabular}


Plus récente et destinée à un niveau plus avancé (A2), la méthode Quartier d'affaires (désormais QA) adopte la même structure : les tâches pédagogiques et méta-communicatives se succèdent pour faire ressortir et aborder les contenus de la langue et du travail propres au contexte situationnel décrit dans les unités. En revanche, la tâche cible est déplacée dans la partie finale de la méthode, celleci regroupant au total dix tâches. L'objectif de ces dernières est de permettre aux apprenants « de mettre en pratique les acquis dans des activités réalistes et réalisables, avec des objectifs clairement énoncés, et qui encouragent les travaux en groupe » (QA 2013:3).

L'unité que nous avons choisie pour notre analyse porte le titre « Mon premier jour dans l'entreprise ». L'ensemble des tâches qu' elle propose visent aux objectifs communicationnels (professionnels) et à préparer l'apprenant à décrire son lieu de travail, présenter l'organigramme d'une entreprise, comprendre le règlement et prendre connaissance des différents types d'entreprises. L'atteinte des objectifs de communication passe par l'appropriation des éléments linguistiques lexicaux (lieux de travail, types d'entreprises) et grammaticaux (impératif affirmatif et négatif, il faut + infinitif, complément du nom) (QA 2013 : 39). La tâche finale qui couronne l'activité des apprenants leur demande de « réaliser une présentation claire de [leur] lieu de travail ou (d'études), [...] présenter l'organisation générale » à l'aide de Power Point, tout ceci à l'attention d'une importante personnalité francophone qui vient visiter l'entreprise (QA 2013 : 118).

Les activités langagières requises pour la résolution de la tâche finale (celle-ci correspondant parfaitement à une activité professionnelle courante) contraignent les apprenants tout d'abord à lire / écouter et comprendre les textes / discours exploités dans le cadre des tâches pédagogiques de communication afin de repérer les éléments de savoirs et savoir-faire communicatifs et professionnels. Lors du traitement des textes / discours, les apprenants focalisent leur attention sur les aspects organisationnels du travail dans l'entreprise (types d'entreprises, professions, postes, fonctions, relations hiérarchiques, règles internes d'une entreprise) tout en effectuant les activités langagières relatives :

- à la compréhension des messages («Écoutez le dialogue / les témoignages / l'interview et répondez » (QA 2013 : 40-41, 44),

- à la production des textes ("Représentez l'organigramme du service marketing de Sant\&Pharm » (QA 2013: 42), «Y a-t-il des différences avec la réglementation de votre pays ? Lesquelles ? Qu' est-ce qu'il ne faut pas faire dans les entreprises de votre pays? (QA 2013 : 43), 
- à l'interaction («Un(e) ami(e), ou votre famille, vous interroge sur votre premier jour en entreprise. Jouez la scène à deux» (QA 2013 : 40), «Vous avez créé une entreprise dans votre pays et vous préparez le règlement destiné au service informatique. Imaginez 5 droits et / ou 5 interdictions » (QA $2013: 43$ ),

- à la médiation («Quel type d'entreprise correspond à chaque situation ? Justifiez votre choix » (QA 2013 : 44).

Les tâches pédagogiques s'enchaînent avec les tâches méta-communicatives qui fournissent les ressources linguistiques (lexique relatif au personnel, à la structure de l'entreprise et aux lieux de travail, aux qualités du personnel, aux droits et interdictions, aux types d'entreprises etc.) et contribuent à la stabilisation de ces dernières dans le répertoire langagier des apprenants (« Mettez les phrases à la forme impérative »(QA $2013: 41)$, « Faites des phrases selon le modèle avec les mots proposés » (QA $2013: 43)$, « Complétez la phrase avec la préposition qui convient» (QA $2013: 45)$.

Toutes ces composantes sont finalement réinvesties dans la résolution de la tâche finale, celle-ci prescrivant minutieusement le travail à effectuer :

1. Vous faites un plan simple et clair des lieux : différents services, entrées de secours, ascenseurs, escaliers, toilettes [...]

2. Vous faites un organigramme clair. Vous pouvez vous aider d'un logiciel (outil de création de cartes mentales, par exemple). Vous pouvez ajouter à l'organigramme des photos, le logo de l'entreprise ou de centre de formation, des illustrations [...]

3. Vous rédigez des textes pour accompagner la présentation. Il y a deux types de textes : les textes écrits pour accompagner les diapositives et les textes à dire à l'oral pendant la présentation.

4. Vous mettez en forme le document final : une présentation Power Point.

5. Vous présentez votre travail à la classe (QA 2013 : 118).

La tâche complexe active de par sa nature diverses activités langagières effectuées à des étapes successives du travail dans le cadre desquelles sont combinés les items linguistiques conformément aux règles du système linguistique. De plus, elle sensibilise les apprenants aux aspects professionnels de la communication au travail face à un groupe : choix du support, organisation de la présentation, rédaction des diapositives, message à transmettre sous forme écrite (contenu des diapos) et orale (commentaire oral sur les diapos).

Voici, la mise en commun synthétique des éléments intégrés dans l'unité examinée à travers les tâches mises en place : 
Tableau 2. «Mon premier jour dans l'entreprise » $(\mathrm{QA})$ : intégration des savoirs et savoir-faire langagiers et professionnels

\begin{tabular}{|c|c|c|c|c|}
\hline \multirow[b]{2}{*}{ Actes de parole } & \multicolumn{2}{|c|}{ Savoirs et savoir-faire linguistiques } & \multirow{2}{*}{$\begin{array}{l}\text { Savoirs profes- } \\
\text { sionnels }\end{array}$} & \multirow{2}{*}{$\begin{array}{c}\text { Savoir-faire } \\
\text { professionnels / } \\
\text { communication- } \\
\text { nels }\end{array}$} \\
\hline & Lexique & Grammaire & & \\
\hline $\begin{array}{l}\text { 1. Décrire son } \\
\text { lieu de travail }\end{array}$ & \multirow{3}{*}{$\begin{array}{l}\text { - intitulés de } \\
\text { poste, profes- } \\
\text { sions ; } \\
\text { - relations hié- } \\
\text { rarchiques ; } \\
\text { - lieux de tra- } \\
\text { vail, noms de } \\
\text { départements, } \\
\text { fonctions ; } \\
\text { - objets et pro- } \\
\text { duits ; } \\
\text { - qualités du } \\
\text { personnel ; } \\
\text { - droits et obli- } \\
\text { gations ; } \\
\text { - types } \\
\text { d'entreprises. }\end{array}$} & \begin{tabular}{|l} 
- complément \\
du nom avec \\
les préposi- \\
tions de, en, \\
à, p.ex. bureau
\end{tabular} & \multirow{3}{*}{\begin{tabular}{|l} 
- connaître \\
la structure \\
organisation- \\
nelle ; \\
- connaître et \\
situer diffé- \\
rents lieux de \\
travail, avoir \\
conscience de \\
leur rôle ; \\
- connaître diffé- \\
rentes formes \\
de sociétés \\
(critères : taille, \\
statut ju- \\
ridique) ; \\
- connaître les \\
droits et les \\
obligations des \\
salariés ; \\
- identifier \\
l'activité (pro- \\
duits et ser- \\
vices).
\end{tabular}} & \multirow{3}{*}{$\begin{array}{l}\text { - } \text { identifier les } \\
\text { lieux de travail, } \\
\text { les décrire } \\
\text { (fonction, équi- } \\
\text { pement); } \\
\text { - expliquer } \\
\text { la place des } \\
\text { salariés dans la } \\
\text { structure or- } \\
\text { ganisationnelle } \\
\text { et les relations } \\
\text { hiérarchiques ; } \\
\text { - identifier et } \\
\text { comprendre } \\
\text { les comporte- } \\
\text { ments (in)ac- } \\
\text { ceptables au } \\
\text { travail : les } \\
\text { ordonner et } \\
\text { interdire ; } \\
\text { - présenter son } \\
\text { entreprise } \\
\text { à l'oral à l'aide } \\
\text { d'un support } \\
\text { visuel (Power } \\
\text { Point). }\end{array}$} \\
\hline $\begin{array}{l}\text { 2. Présenter / } \\
\text { expliquer les } \\
\text { relations hié- } \\
\text { rarchiques }\end{array}$ & & $\begin{array}{l}\text { de l'ingénieur, } \\
\text { salle de ré- } \\
\text { union, chaise } \\
\text { à roulettes, } \\
\text { bureau en bois }\end{array}$ & & \\
\hline $\begin{array}{l}\text { 3. Ordonner vs } \\
\text { interdire }\end{array}$ & & 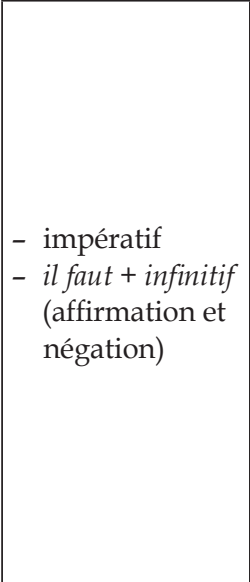 & & \\
\hline
\end{tabular}

La troisième des méthodes de FOS choisies Objectif Express (désormais OE), de niveau $\mathrm{A} 1>\mathrm{A} 2$, aborde également le sujet de présentation professionnelle dans le cadre de l'unité intitulée « Présentez une entreprise !» (OE 2013 : 109-123). Les objectifs énoncés pour la séquence visent à rendre l'apprenant capable « de parler d'une entreprise et de son organisation, de comprendre et de donner des explications simples sur un processus de fabrication, de comprendre et de rédiger un règlement simple » (OE 2013 : 109). En termes d'objectifs communicatifs (qui font par ailleurs partie intégrante des objectifs professionnels), les buts se décomposent en capacités de décrire une entreprise (par rapport à son historique, sa situation, son organisation, son activité et les chiffres-clés), de présenter le personnel de l'entreprise (du point de vue des qualités personnelles et professionnelles), d'expliciter la composition d'un produit et le processus de 
sa fabrication (par rapport à la chronologie des actions et leur durée), d'exprimer l'obligation et l'interdiction dans un règlement ainsi que de préciser un cas particulier dans ce règlement. A cette fin, l'unité en question met en place une gamme de tâches pédagogiques dont le but est de pratiquer les activités de compréhension [à partir de l'enregistrement «Complétez la carte d'identité de l'entreprise »(OE 2013 : 110), « Lisez la carte d'identité et répondez aux questions » (OE $2013: 111)$ ] et de production [« Rédigez une courte présentation de chaque entreprise » (OE Cahier d'activités $2013: 69$ ), « Présentez une personne très connue de votre choix » (OE Cahier d'activités $2013: 70)$, « Rédigez une fiche technique pour expliquer le processus de fabrication d'un objet de verre » (OE Cahier d'activités $2013: 73)$ ].

Les tâches de communication sont étroitement liées aux exercices de langue abordant des questions particulières de la langue, comme le présentatif $c^{\prime} e s t$, le graduatif très, le pronom personnel indéfini on, les pronoms relatifs simples qui / que, la forme active et la voix passive, la conjugaison des verbes produire, construire, vendre, mettre, tenir.

L'enchaînement des diverses tâches à accomplir est strictement subordonné à la structure organisationnelle de l'unité. Cette dernière se décompose en quatre étapes (A-D) dont l'organisation passe toujours par quatre points majeurs : «Écoutez / Lisez », « Réagissez », «Retenez », « Passez à l'action ». La méthode met ainsi en place un scénario fixe allant des tâches de réception (orales ou écrites) sensibilisant les apprenants aux aspects linguistiques et professionnels par les tâches méta-communicatives abordant les problèmes de la langue aux tâches de production orale et écrite. Ces dernières interviennent toujours à la fin des chacune des étapes A-D dans la rubrique « Passez à l'action » et amènent les apprenants à intervenir à l'intermédiaire de la langue par rapport à un problème concret inséré dans un contexte professionnel :

A.1. Vous avez un poste important chez Boisjoli et vous avez un entretien avec un(e) journaliste. Répondez à ses questions (indiquez des événements-clés, la catégorie de l'entreprise et sa situation géographique ; décrivez les activités principales ; donnez des chiffres-clés) (OE 2013 : 111).

A.2. Votre entreprise va participer à un salon en France. Rédigez un petit texte pour la présenter dans une brochure que vous allez donner aux visiteurs de votre stand (OE 2013 : 111).

B.1. Vous travaillez dans l'entreprise Simax. Présentez le service commercial de cette entreprise (OE 2013 : 113).

B.2. Faites l'organigramme du service ou de l'entreprise où vous travaillez. Présentez-le oralement à des partenaires (OE 2013 : 113).

C.1. Vous faites visiter une conserverie à des clients francophones. Décrivez le processus de fabrication des boîtes de conserve de haricots verts (OE 2013 : 115). 
La tâche pédagogique au service du développement des savoir-faire langagiers et professionnels 173

C.2. Vous travaillez dans une usine. Vous êtes chargé de rédiger la fiche technique du procédé de fabrication du produit fabriqué dans cette usine. Choisissez un produit et rédigez la fiche.

D.1. Choisissez un lieu public (restaurant, hôpital, musée, etc.) et rédigez un règlement pour les visiteurs (OE 2013 : 117).

La diversité de tâches, que l'unité en question met en place, prépare les apprenants aux différentes situations de travail où ils seront amenés à agir avec la langue à l'écrit et à l'oral lors de l'exécution de leurs diverses responsabilités professionnelles. La combinaison des ressources linguistiques investies dans les textes / discours mis au service des tâches de communication permet aux apprenants de s'approprier les savoirs et savoir-faire requis dans leur future activité professionnelle.

Tableau 3. « Présentez une entreprise » (OE) : intégration des savoirs et savoir-faire langagiers et professionnels

\begin{tabular}{|c|c|c|c|c|}
\hline \multirow{2}{*}{$\begin{array}{c}\text { Actes de } \\
\text { parole }\end{array}$} & \multicolumn{2}{|c|}{ Savoirs et savoir-faire linguistiques } & \multirow{2}{*}{\begin{tabular}{|c|} 
Savoirs \\
professionnels
\end{tabular}} & \multirow{2}{*}{$\begin{array}{l}\text { Savoir-faire pro- } \\
\text { fessionnels / com- } \\
\text { municationnels }\end{array}$} \\
\hline & Lexique & Grammaire & & \\
\hline $\begin{array}{l}\text { 1. Parler de } \\
\text { son entre- } \\
\text { prise }\end{array}$ & $\begin{array}{l}\text { - les catégories de } \\
\text { l'entreprise : PME, } \\
\text { entreprise familiale, } \\
\text { groupe..., } \\
\text { - les commerces et les } \\
\text { commerçants (bouche- } \\
\text { rie boucher...), } \\
\text { - les points cardinaux } \\
\text { (nord sud est ouest), } \\
\text { - les chiffres, } \\
\text { - verbes produire, fabri- } \\
\text { quer, construire, créer, } \\
\text { exporter, commercialiser, } \\
\text { vendre, développer... }\end{array}$ & 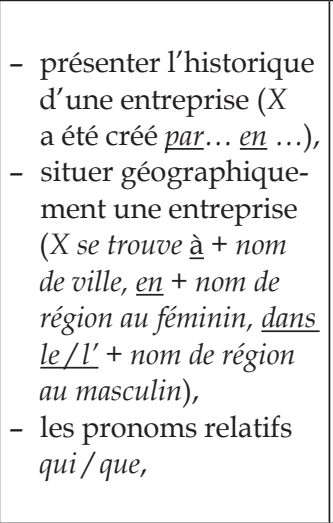 & \begin{tabular}{|l} 
- connaître \\
différentes \\
catégories \\
d'entreprises, \\
- connaître \\
différentes \\
activités des \\
entreprises,
\end{tabular} & $\begin{array}{l}\text { - présenter } \\
\text { l'activité de } \\
\text { l'entreprise, sa } \\
\text { taille, sa struc- } \\
\text { ture, } \\
\text { - situer } \\
\text { géographique- } \\
\text { ment une entre- } \\
\text { prise, } \\
\text { - décrire } \\
\text { l'organisation } \\
\text { d'une entreprise, }\end{array}$ \\
\hline $\begin{array}{l}\text { 2. Présenter } \\
\text { le person- } \\
\text { nel de } \\
\text { l'entreprise }\end{array}$ & $\begin{array}{l}\text { - les services et fonctions } \\
\text { dans une entreprise, } \\
\text { - les relations hié- } \\
\text { rarchiques, } \\
\text { - les qualités person- } \\
\text { nelles et profession- } \\
\text { nelles. }\end{array}$ & - le présentatif c'est, & $\begin{array}{l}- \text { connaître les } \\
\text { fonctions des } \\
\text { salariés et } \\
\text { leur emplace- } \\
\text { ment dans la } \\
\text { structure de } \\
\text { l'entreprise, }\end{array}$ & $\begin{array}{l}\text { - présenter le } \\
\text { personnel, } \\
\text { - dessiner } \\
\text { l'organigramme } \\
\text { et expliquer } \\
\text { la place des } \\
\text { salariés dans la } \\
\text { structure or- } \\
\text { ganisationnelle } \\
\text { de l'entreprise } \\
\text { et les relations } \\
\text { hiérarchiques } \\
\text { entre eux, }\end{array}$ \\
\hline
\end{tabular}




\begin{tabular}{|c|c|c|c|c|}
\hline \multirow{2}{*}{$\begin{array}{l}\text { Actes de } \\
\text { parole }\end{array}$} & \multicolumn{2}{|c|}{ Savoirs et savoir-faire linguistiques } & \multirow{2}{*}{\begin{tabular}{c|} 
Savoirs \\
professionnels
\end{tabular}} & \multirow{2}{*}{$\begin{array}{l}\text { Savoir-faire pro- } \\
\text { fessionnels / com- } \\
\text { municationnels }\end{array}$} \\
\hline & Lexique & Grammaire & & \\
\hline $\begin{array}{l}\text { 3. Décrire le } \\
\text { processus } \\
\text { de fabrica- } \\
\text { tion }\end{array}$ & $\begin{array}{l}\text { - les types de produits, } \\
\text { matières, emballages, } \\
\text { - les verbes pour décrire } \\
\text { le processus : chauffer, } \\
\text { cuire, mélanger, ajouter... }\end{array}$ & $\begin{array}{l}\text { - la voix passive (être + } \\
\text { participe passé), } \\
\text { - situer dans le temps } \\
\text { (la chronologie), } \\
\text { - exprimer la durée, } \\
\text { - indiquer la composi- } \\
\text { tion d'un produit : se } \\
\text { compose de est composé } \\
\text { de..., }\end{array}$ & $\begin{array}{l}\text { - connaître les } \\
\text { produits de } \\
\text { l'entreprise et } \\
\text { les procédés } \\
\text { de fabrication, }\end{array}$ & $\begin{array}{l}\text { - } \text { expliciter le } \\
\text { processus de } \\
\text { fabrication, } \\
\text { - rédiger la fiche } \\
\text { technique, }\end{array}$ \\
\hline $\begin{array}{l}\text { 4. Ordonner } \\
\text { vs interdire }\end{array}$ & $\begin{array}{l}\text { - le personnel de } \\
\text { l'entreprise }\end{array}$ & $\begin{array}{l}\text { - exprimer } \\
\text { l'obligation : il } \\
\text { faut impérativement, } \\
\text { devoir, est/sont } \\
\text { obligatoire(s)..., } \\
\text { - exprimer } \\
\text { l'interdiction (il est } \\
\text { interdit, il ne faut pas, } \\
\text { ne pas pouvoir, est } \\
\text { interdit...), } \\
\text { - indiquer une excep- } \\
\text { tion ou un cas par- } \\
\text { ticulier (en cas de...). }\end{array}$ & $\begin{array}{l}\text { - connaître les } \\
\text { droits et les } \\
\text { obligations } \\
\text { des salariés. }\end{array}$ & $\begin{array}{l}\text { - } \text { identifier et } \\
\text { comprendre les } \\
\text { comportements } \\
\text { (in)acceptables } \\
\text { dans une entre- } \\
\text { prise : les ordon- } \\
\text { ner et interdire, } \\
\text { - rédiger un rè- } \\
\text { glement interne. }\end{array}$ \\
\hline
\end{tabular}

\section{EN GUISE DE CONCLUSION}

L'examen des tâches des trois méthodes de FOS que nous avons effectué ci-avant nous amène à constater que le caractère actionnel des activités pédagogiques rend possible l'intégration de contenus de diverse nature : linguistiques, communicatifs, professionnels. Il convient de souligner une forte interrelation entre tous les éléments enseignés grâce à un agencement raisonnable des tâches mises en place. L'appropriation des ressources de la langue ainsi que le perfectionnement des activités langagières entretiennent toujours un lien explicite avec les objectifs de communication professionnelle et le contexte du travail de référence. L'apprenant est alors capable d'établir des corrélations entre les contenus d'apprentissage et l'utilité de ces derniers dans les actions langagières qu'il accomplira ultérieurement au travail : il sait ce qu'il apprend et pourquoi il l'apprend parce que la tâche finale donne toute leur pertinence aux moyens langagiers investis au profit des actions convenues dans le milieu du travail.

De plus, même à partir des trois unités des méthodes de FOS distinctes, il est possible d'observer la mise en place de différentes démarches pour aborder le 
sujet de présentation en milieu professionnel. L'accueil en contexte professionnel diffère certainement de la présentation (formelle ou informelle) au quotidien et contient des données censées révéler le lieu et la nature du travail effectué. En outre, se présenter au travail centre l'attention non seulement sur la personne qui se présente, mais aussi sur l'établissement employeur. Et, selon le cas, la présentation de ce dernier peut comporter des éléments plus ou moins détaillés allant des données officielles standard (la raison sociale, la forme juridique, la taille, l'activité exercée) par la présentation du personnel (la structure organisationnelle et les relations hiérarchiques) jusqu'à la définition des normes en vigueur au travail et / ou la description des produits et les processus de fabrication. Un tel éventail de tâches résolues dans les situations de présentation et les éléments que celle-ci peut comporter contraint à se rendre compte de la complexité des savoirs et savoir-faire requis. Ces derniers exigent assurément des connaissances linguistiques, mais la mobilisation opérationnelle de celles-ci est étroitement conditionnée par les circonstances de l'action langagière (qui ? où ? à qui ? quand ? pourquoi ? comment ?) qui, de son côté, met en scène des scénarios interactionnels variés et recourt aux textes / discours particuliers.

Les méthodes de FOS analysées montrent que, en fonction de la situation dans laquelle la présentation a lieu, l'acte de se présenter peut se dérouler de manière différente tout en orientant l'attention des interlocuteurs sur les éléments contextuels qui conditionnent à la fois l'action et l'emploi des ressources langagières. Étant donné que c'est le contexte qui suscite la parole dans l'activité, il convient à l'acteur social de sélectionner les contenus à mettre en avant et de décider de la nature des éléments langagiers à mobiliser. La sensibilisation à la variabilité des situations professionnelles au cours de l'apprentissage aide l'apprenant à effectuer ses choix linguistiques avec plus de conscience et - par conséquent - de chances de réussite, ce que nous avons vu avec l'exemple des tâches de communication proposées par les méthodes analysées. Dans bien des cas, le faire équivaut au dire ce dont les tâches examinées rendent compte sous forme des discours ou écrits professionnels (comme p.ex. le règlement interne, la fiche technique, la présentation multimédia) où la parole intervient comme activité principale. Enfin, sont également présentes les tâches qui contraignent l'apprenant à réfléchir sur l'action effectuée dans l'entreprise. Nous pouvons y classer les activités visant à expliciter les processus de fabrication d'un produit (la nature des actions effectuées et leur agencement chronologique) ou la réflexion sur les comportements (in)adaptés aux normes de l'entreprise (les interdictions et les ordres). Du fait que les méthodes retenues pour notre analyse s'adressent à un niveau élémentaire, nous constatons qu'il est possible de sensibiliser les apprenants aux différents statuts de la langue au travail dès le début de leur apprentissage. 


\section{RÉFÉRENCES}

Bronckart, J.-P. (2001). S'entendre pour agir et agir pour s'entendre. In: J. M. Baudouin / J. Friedrich (dir.). Théories de l'action et éducation (pp. 133-154). Bruxelles: De Boeck.

Conseil de l'Europe (2001). Cadre Européen Commun de Référence pour les Langues. Apprendre, Enseigner, Évaluer. Paris: Didier.

De Terssac, G. (1996). Savoirs, compétences et travail. In: J. M. Barbier (dir.). Savoirs théoriques et savoirs d'action (pp. 223-248). Paris: PUF.

Devitt, S. (2002). Guide à l'usage des apprenants et des enseignants. In: J. L. M. Trim. (dir.). Cadre européen commun de référence pour les langues: apprendre, enseigner, évaluer. Guide pour les utilisateurs (pp. 83-116). Strasbourg: Conseil de l'Europe, Division des Politiques Linguistiques.

Halté, J. F. (1982). Apprendre autrement à l'école. Pratiques, 36, 5-23.

Lacoste, M. (2001). Peut-on travailler sans communiquer? In: A. Borzeix / B. Fraenkel (dir.). Langage et travail (pp. 21-54). Paris: CNRS Éditions.

Mangiante, J.-M. / Parpette, Ch. (2004). Le Français sur Objectif Spécifique: de l'analyse des besoins à l'élaboration d'un cours. Paris: Hachette.

Narcy-Combes, J.-P. / Narcy-Combes, M.-F. (2007). La tâche, un moyen pour optimiser l'enseignement apprentissage de l'anglais aux spécialistes d'autres disciplines dans le contexte universitaire français. Le Français dans le Monde. Recherches et Applications, 42, 73-86.

Sowa, M. (2011). D'une activité pédagogique à l'activité professionnelle. Le cheminement vers la compétence. Lublin: TN KUL.

Received: 19.07.2019; revised: 21.03 .2020

\section{MAGDALENA SOWA}

Uniwersytet Marii Curie-Skłodowskiej w Lublinie

magdalena.sowa@umcs.pl

ORCID: 0000-0002-9571-8693 